\title{
A Precision Power Amplifier for Power/Energy Calibration Applications
}

\author{
OWEN B. LAUG, MEMBER, IEEE
}

\begin{abstract}
A precision power amplifier for use in power/energy calibration applications is described. The amplifier was primarily designed to boost the output amplitude of a digital generator to provide the nominal 120- or 240 -rms voltage component of a "phantom" calibration power source. The amplifier has a fixed gain of 40 and can provide a maximum output voltage swing of $970 \mathrm{~V}$ peak-to-peak or $340-\mathrm{V}$ rms at $100-\mathrm{mA}$ rms. The bandwidth is from dc to $150 \mathrm{kHz}$ and at $60 \mathrm{~Hz}$ the observed no-load short-term amplitude and phase instabilities are $\pm 5 \mathrm{ppm}$ and $\pm 5 \mu \mathrm{rad}$, respectively. The amplifier design uses high-voltage $N$-channel MOSFET's in the output driver stage together with a unique circuit topology of opto-isolators between the lowlevel input stage and the high-level output stage.
\end{abstract}

\section{INTRODUCTION}

A PRECISION high-voltage power amplifier that has been developed as part of a digitally synthesized source of "phantom" power for calibrating electrical power and energy meters is described. The power amplifier is used to boost the output of one channel of a dualchannel generator to a nominal 120 - or $240-\mathrm{V}$ rms [1]. In addition, the amplifier was designed to supply up to 100-mA rms to accommodate the burden requirements of electrodynamic-type meters without causing significant error. A prime goal was to maintain the excellent shortterm amplitude and phase stability inherent in the digital generator. In addition to the above specific application, there is a general need for a precision power amplifier that will provide an output swing of several hundred volts at moderate power levels while maintaining good dc characteristics, stable gain, and wide bandwidth.

General purpose commercial integrated circuit and hybrid power operational amplifiers partially fill this need with typical output swings up to $\pm 145 \mathrm{~V}$ peak with currents as high as $75 \mathrm{~mA}$ peak. Their maximum output voltage swing is limited by the safe-operating voltage ratings of the complimentary output driver transistors employed in the amplifiers. Another approach to achieving the accuracy of a low-voltage operational amplifier with highvoltage output swings is to employ a separate voltage booster stage to provide the additional voltage gain and high output swings. Several unique high-voltage booster circuits designed to enhance the output levels of low-voltage operational amplifiers have been described in the lit-

Manuscript received April 28, 1987; revised June 20, 1987.

The author is with the National Bureau of Standards, Building 220, Room B162, Gaithersburg, MD 20899.

IEEE Log Number 8716876. erature [2]-[6]. However, most of these booster schemes depend on complimentary devices which limit the maximum output voltage to the device with the lowest safe operating voltage rating (usually $P$-type semiconductor devices). Often a booster stage is enclosed in the operational amplifier feedback loop so that the effects of gain and offset errors are reduced by the loop gain of the system. However, connecting a voltage booster stage to the output of a low-voltage operational amplifier with overall feedback requires careful consideration of the dynamic parameters such as bandwidth, phase shift, and slew rate in order to reduce the likelihood of oscillation. The design and application of a voltage booster stage coupled to a high-gain operational amplifier (while maintaining precise closed-loop gain at high output swings and wide bandwidth) is difficult to achieve.

The performance of an output voltage booster stage is highly dependent of the characteristics of the semiconductor devices. Recent improvements in metal oxide semiconductor (MOS) technology has made available moderate power level $N$-channel MOSFET's with voltage ratings of up to $1000 \mathrm{~V}$ with continuous currents of about $3 \mathrm{~A}$ and dissipation ratings up to $75 \mathrm{~W}$. More importantly, for a given voltage rating, MOSFET's tend to have a greater safe-operating region that is not limited by the second breakdown failure phenomena which plagues bipolar transistors (BJT's). Thus MOSFET's do not need an elaborate protection scheme to control the voltage/current locus to a particular restrictive safe operating region. This becomes an important practical consideration for linear operation. Other desirable characteristics of MOSFET's such as their low drive power requirements and inherently wide bandwidth make them an ideal choice for linear highvoltage driver circuits. Unfortunately, similar to BJT's, the unavailability of complimentary $(P$-channel) MOSFET's with high-voltage ratings comparable to their $N$-channel counterpart, limits the maximum peak-to-peak output swing to about $500 \mathrm{~V}$ in a class B complimentary symmetry output driver configuration. Despite this limitation it is still possible to configure a class $\mathrm{B}$, direct coupled, bipolar output driver stage using a pair of high-voltage $N$-channel MOSFET's in a stacked arrangement. Although such a configuration tends to complicate the bias and drive circuitry, the amplifier presented in this paper utilizes a unique opto-isolator coupled output driver system that can be driven by a low-voltage operational amplifier. The particular design of the output stage over- 
comes some of the past difficulties in maintaining a stable bias current in a MOSFET, Class B output driver [7].

\section{General Design Approach}

Fig. 1 shows the conceptual diagram of a circuit topology which forms the basis of the output driver of the power amplifier. The circuit consists of two $\mathrm{N}$-channel, power MOSFET devices each connected as a variable current source and series-connected between positive and negative supply voltages. If each resistor is varied in proportion to alternate positive and negative half-cycle signals then the summation of the currents in the load will result in a reconstruction at the output of the entire bipolar signal. Such an arrangement of output devices is often referred to as a Class B totem pole driver. By adjusting the resistors so that a small equal current flows through both devices the result will be a zero output voltage at the load. Biasing each device with a small idling or quiescent current is usually done for Class B output drivers as a practical measure to avoid the nonlinearities and attendant crossover distortion associated with devices operating near cutoff.

The most practical way of effecting a resistance or conductance change in proportion to a signal as suggested in Fig. 1 is to replace each of the resistors with a second MOSFET which acts as the current controlling device. The resulting configuration (shown in Fig. 2) can be recognized as a stacked pair of cascode stages (common source stage driving a common base stage). Another way of viewing the configuration in Fig. 2 is as two seriesconnected, controllable current sources, driving a common load with compliance voltages approaching the respective positive and negative supply voltages. This configuration has a number of advantages and one disadvantage. First, from a frequency-response point of view the cascode configuration reduces the Miller effect capacitances associated with the devices and, hence, wider bandwidths are possible. Also, each of the current controlling devices, $Q 2$ and $Q 4$ can be relatively low-voltage transistors operating with a drain-to-source voltage of $\left(V_{B}\right.$ $-V_{T}$ ) where $V_{T}$ is the gate-to-source threshold voltage of either $Q 1$ or $Q 3$. The common-mode voltage of the input control signal for the lower driver is constant and equal to the negative supply voltage. The common-mode voltage for the upper driver, however, is not constant but equal to the dynamic peak-to-peak output signal. The main disadvantage here is that large output voltage swings with high slew rates impose a very stringent design requirement on the high frequency, common-mode rejection properties of the upper drive circuit. This difficult requirement was met with a differential, optically isolated coupling system.

Fig. 3 shows an elaboration of the output driver stage of Fig. 2 included with a simplified schematic of the entire amplifier system. The battery potentials, $V_{B}$, of Fig. 2 have been replaced with $12-\mathrm{V}$ reference diodes each biased by resistors to the positive and negative power supplies. An additional fixed resistor, $R_{S}$, in each driver pair

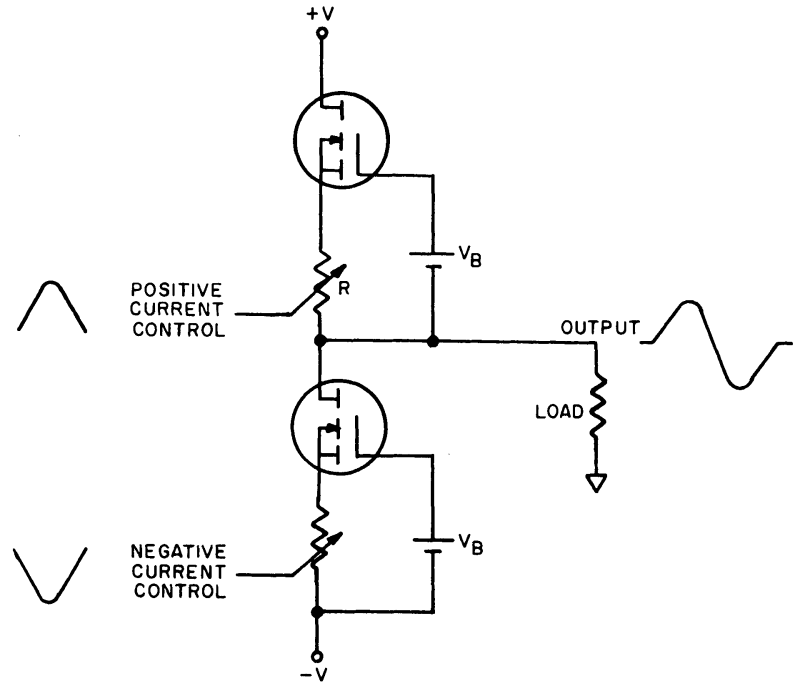

Fig. 1. Conceptual diagram of the power output stage.

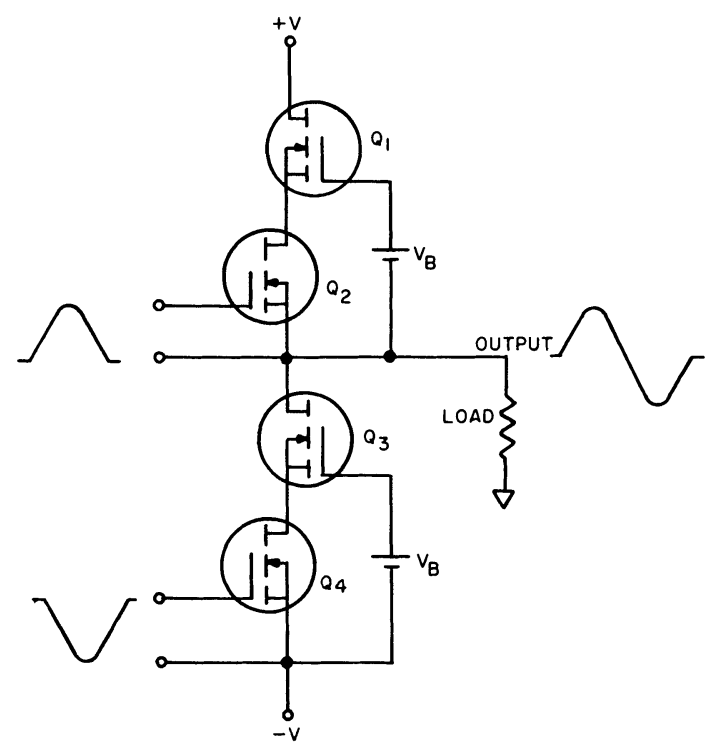

Fig. 2. A practical embodiment of the power output stage shown in Fig. 1 .

serves a dual purpose. It is part of a local feedback system to stabilize the gain and quiescent bias current of each driver, and in conjunction with an overload circuit it limits the maximum current that can be delivered to the load as follows: As the load current increases the voltage drop across $R_{s}$ rises until the drain-to-source voltage of $Q 2$ or $Q 4$ reaches saturation, at which point the gain drops sharply. The output voltage of $U 1$ rises to try to overcome the loss in gain, and a bistable overload sensing circuit senses the increased voltage and disconnects the supply voltages from the output drivers.

A polarity separator circuit at the output of amplifier $U 1$ separates the signal into positive and negative components and steers the respective polarized signals to two dual opto-isolators. The two dual opto-isolators couple the polarized analog signals in a differential manner across the large common-mode voltage between the low-level input circuit and the high-level output circuit. The output 


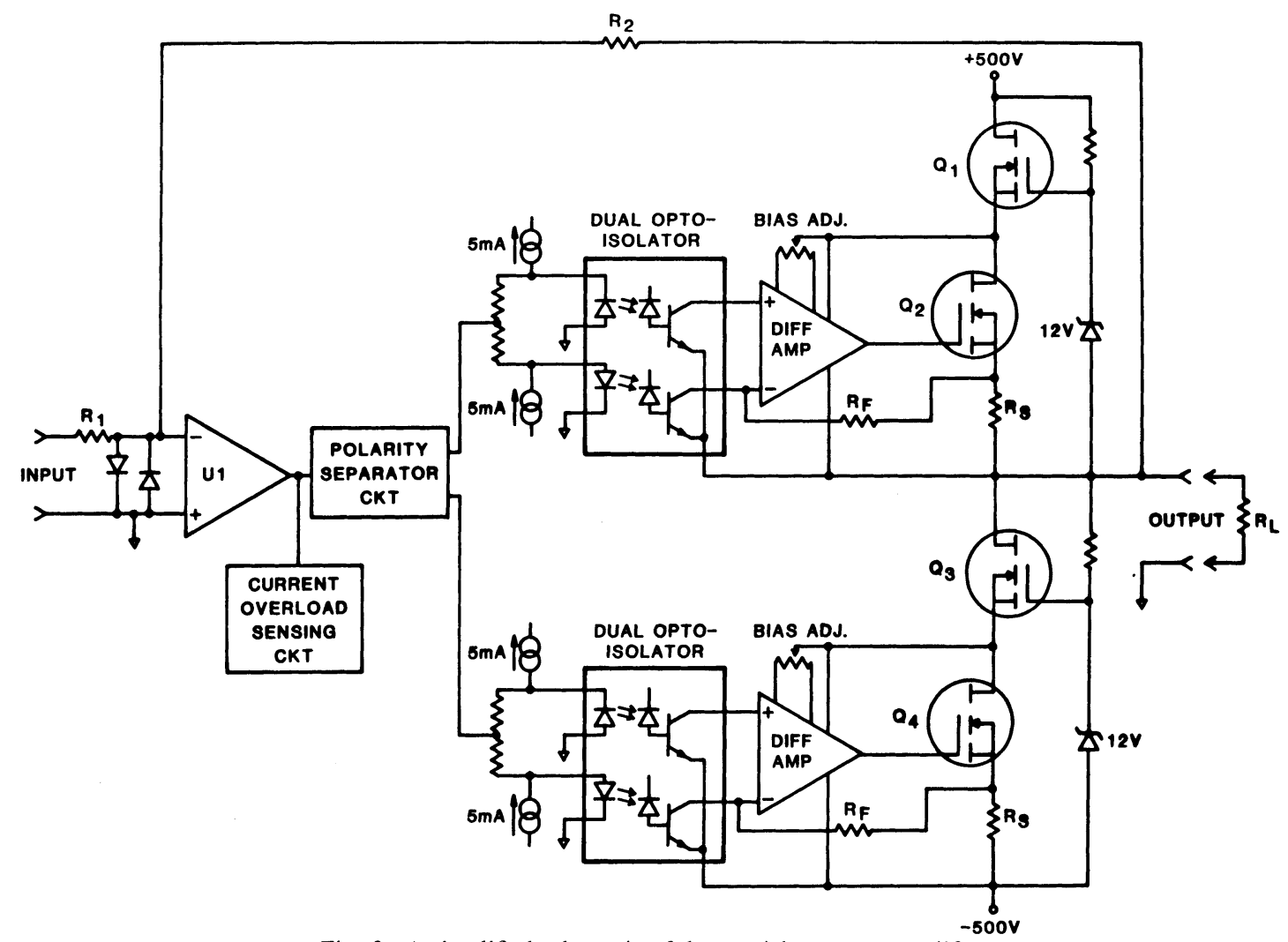

Fig. 3. A simplified schematic of the precision power amplifier.

voltage across the load resistor is fed back through $R 2$ where it is compared with the input voltage from $R 1$ at the summing junction of $U 1$. Thus with sufficient openloop gain, the closed-loop gain of the entire power amplifier becomes operationally equal to $-(R 2 / R 1)$.

Using a pair of opto-isolators in a differential fashion greatly improves the high-frequency common-mode rejection ratio over a single opto-isolator. Insufficient highfrequency common-mode rejection in the upper or positive isolation system can inhibit proper operation. If not sufficiently rejected, high slew rate output signals will result in a current across the small yet finite isolation capacitance between photoemitter and photodetector. This current will either oppose the input drive signal resulting in a delayed turnoff of the drivers, or worse yet, aid the input signal to the point of instability and possible oscillation. A second (and equally important) reason for using dual opto-isolators in a differential fashion is to improve the linearity of the analog transfer function. This improvement is realized for small signals by an approximate cancellation of the gain increment of one opto-isolator against the gain decrement of the second opto-isolator for matched isolators biased at the same operating point on a nonlinear current transfer curve [8]. Each opto-isolator is biased for analog operation so that the photodetector amplifying transistors are neither cut off nor saturated over the operating excursion of the input signal. Also, differential operation tends to improve the dc bias stability of the output stage because temperature and aging effects of the opto-isolator current-transfer ratios will tend to cancel.
The two differential amplifiers shown in Fig. 3 which follow each dual opto-isolator are identical and designed with discrete components. They differ from the usual integrated circuit differential amplifier in that the input is designed to have a low-input impedance which maximizes the bandwidth of the opto-isolators. A bias control on each differential amplifier is used to set the quiescent bias current of each output driver.

\section{Detailed Circuit Description}

Fig. 4 shows a more detailed schematic of one of the identical pair of output drivers, including the opto-isolator and differential amplifier. Each photoemitter diode is forward biased at a quiescent current of about $5 \mathrm{~mA}$ which is established by resistors to the \pm 15 - $\mathrm{V}$ supplies. An input signal voltage applied across terminals 1 and 2 then causes an incremental increase in photoemitter current of one diode and a similar incremental decrease in the second diode. The resultant differential photo-emitter radiant flux is detected, amplified, and converted to a singleended control signal at the gate of $Q 2$.

The particular type of opto-isolator used is critical for this design in order to maximize the high-frequency common-mode rejection, as well as to maximize the normalmode bandwidth. For high-speed operation the photodiode/transistor detector combination is superior to the phototransistor type. The speed of response of a phototransistor detector is inherently slow because the collector-to-base junction must be large in order to capture sufficient photons, hence, a large capacitance. Also, the phototransistor-type isolator tends to have a greater non- 


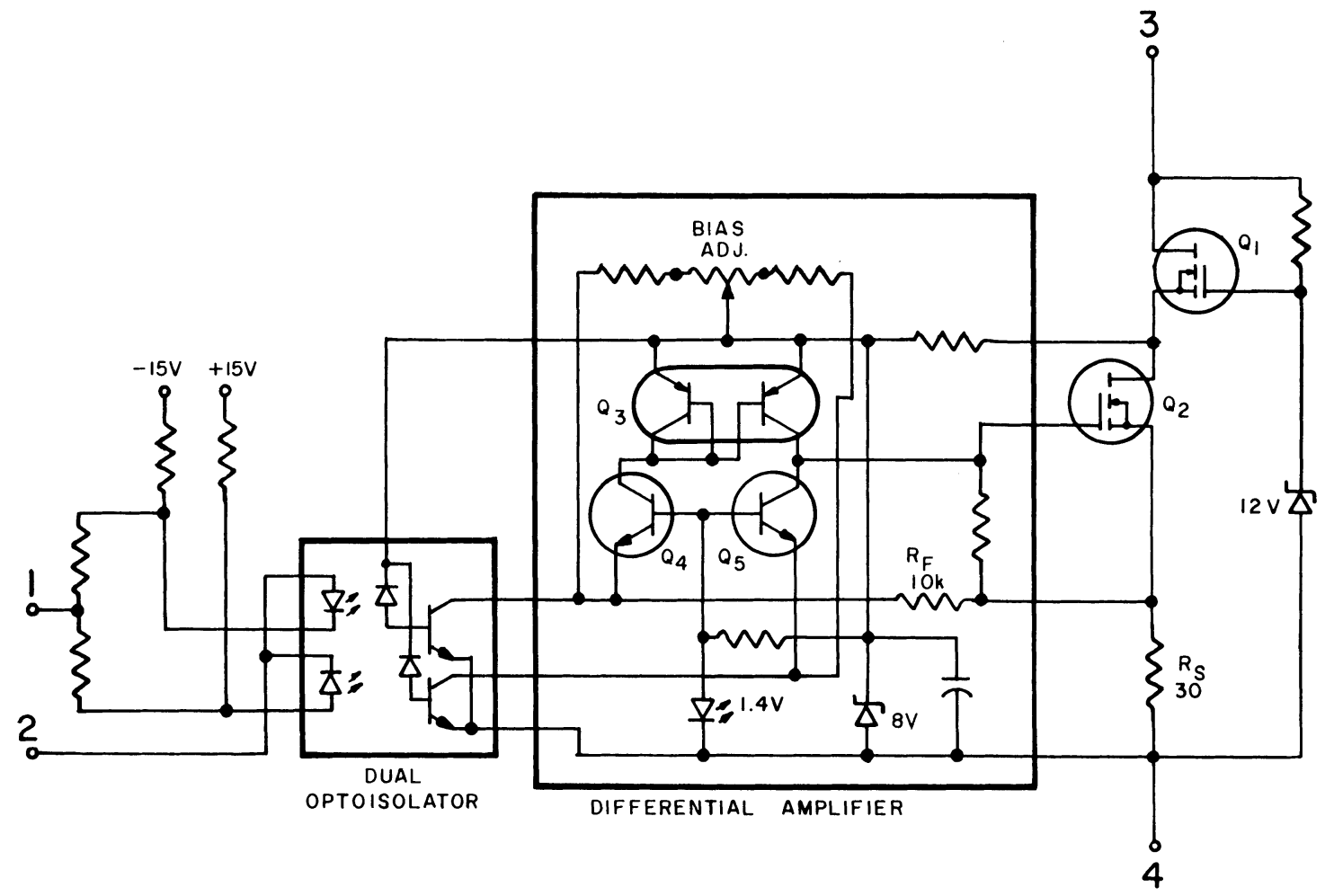

Fig. 4. Detailed schematic of the opto-coupled, output driver.

linearity in its transfer characteristic, thus making it less desirable for analog applications. It is desirable that the photodiode/transistor type of opto-isolators do not have an auxiliary base connection brought out to an external pin. External base connections add additional collectorto-base capacitance, thus reducing bandwidth and increasing the isolation capacitance. The bandwidth of the photodiode/transistor opto-isolator operating in the common emitter configuration (preferred for maximum bandwidth) is an inverse function of the base time constant. An expression for the base time constant $\tau_{b}$, when using a typical photo-diode capacitance of $10 \mathrm{pF}$ and a collector-to-base capacitance $C_{\mathrm{bc}}$ of $0.5 \mathrm{pF}$ operating at $1 \mathrm{~mA}$ of collector current is

$$
\tau_{b} \simeq \beta C_{\mathrm{bc}}\left(R_{L}+525\right)
$$

where $\beta$ is the forward current gain at the $1-\mathrm{mA}$ operating point and $R_{L}$ is the load resistance seen by the collector [8].

From this expression it is clear that maximum bandwidth depends on a minimum $C_{\mathrm{bc}}$ and a low value of load resistance. The limiting factor is the constant term in the expression which is related to the dynamic base resistance. A low value of equivalent load resistance for each collector of the opto-isolator is obtained by driving the emitters of $Q 4$ and $Q 5$ which are connected in a common base configuration. The dynamic input resistance of a common base transistor is inversely proportional to the collector current which at $1 \mathrm{~mA}$ is about $25 \Omega$. This twostage configuration (common emitter driving a common base) again can be recognized as the familiar cascode twostage amplifier which achieves good bandwidth charac- teristics by reducing the Miller effect capacitance. Forward bias for each of the bases of $Q 4$ and $Q 5$ is established by a forward biased LED providing a 1.4-V reference. This reference also serves to establish a collector-to-emitter operating voltage for the photodetector transistors of about $(1.4-0.6)=0.8 \mathrm{~V}$.

The dual-integrated, matched $\mathrm{p}-\mathrm{n}-\mathrm{p}$ transistors, $Q 3$, are connected as a "current mirror" circuit. This circuit functions to "mirror" or transpose the collector current of $Q 4$ into the gate resistor of $Q 2$. The collector current from $Q 5$ flows out of the gate resistor resulting in a summation of $Q 4$ and $Q 5$ collector currents at the gate resistor of $Q 2$. The effect is to produce a "single ended" voltage (common to terminal 4) at the gate of $Q 2$ that is proportional to the differential collector currents of the two photodetector transistors. The gate voltage of $Q 2$ then controls the output current between terminals 3 and 4 . The current gain is stabilized by local negative feedback from the source of $Q 2$ through $R_{F}$ to the emitter of $Q 4$. This feedback reduces the troublesome effect of changes in the output driver quiescent bias current due to temperature and aging of $Q 2$ 's gate threshold voltage. Since the current through $Q 1$ is controlled by $Q 2$, the variable effects of $Q 1$ 's gate threshold voltage do not affect the 'bias stability. In addition to establishing a feedback potential proportional to current at the source of $Q 2, R_{S}$ serves to limit the maximum load current as previously described. Current limiting is set at about $150 \mathrm{~mA}$. The operating supply voltage for the differential amplifier circuit and reverse bias potential for the photo-diodes is derived from the 12$\mathrm{V}$ reference diode minus $Q 1$ 's gate-to-source voltage, and further stabilized by an $8-\mathrm{V}$ shunt regulator diode. The 


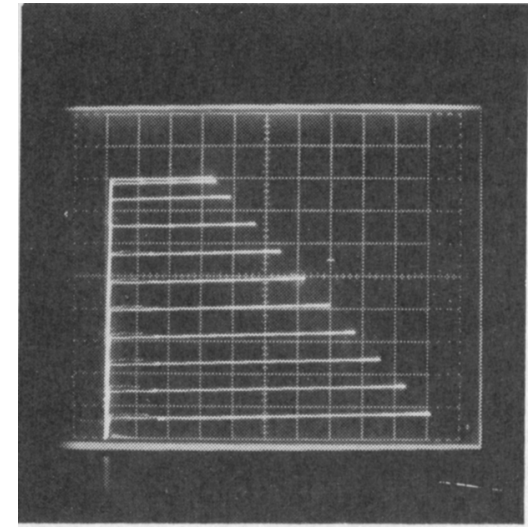

Fig. 5. I/V characteristics of the opto-coupled, output driver (Fig. 4). Horizontal: $100 \mathrm{~V} /$ div; Vertical: $20 \mathrm{~mA} /$ div; Steps: $200 \mu \mathrm{A}$.

bias adjustment potentiometer provides an ajustment for the desired quiescent output current through $Q 1$ and $Q 2$. A bias current of about $15 \mathrm{~mA}$ is sufficient to avoid the nonlinearities in the output driver MOSFET's.

From a model viewpoint the entire circuit of Fig. 4 can be considered as a linear four-terminal device with a current generator across the output terminal pair 3, 4 that is controlled by the current through, or voltage across the input terminal pair 1,2. The model can thus be characterized with a forward current gain or transconductance, an output admittance, and an input impedance. The degree of isolation between input and output can be characterized by the so-called reverse transfer impedance, or simply the isolation capacitance, between output and input terminals. It can be shown that the output current $I_{34}$, assuming linear current transfer ratios of the opto-isolators, an open loop current gain much greater than $R_{F} / R_{S}$, a photoemitter operating current of $5 \mathrm{~mA}$, and neglecting frequency-dependent parameters is

$$
\begin{aligned}
I_{34}= & \left(I_{12}\right)\left(R_{F} / 2 R_{S}\right)\left(K_{1}+K_{2}\right) \\
& +\left(R_{F} / R_{S}\right)\left(K_{1}-K_{2}\right)\left(5 \times 10^{-3}\right)
\end{aligned}
$$

where $K_{1}$ and $K_{2}$ are the forward current transfer ratios of each opto-isolator. Note that because the output circuit is unipolar, $I_{34}$ can only assume values $\geq 0$.

Equation (2) shows that the forward transfer current gain (first term) is proportional to the sum of the individual current transfer ratios, whereas the offset current (second term) is proportional to their differences. Thus the stability of the offset or quiescent current depends on the tracking of the two current transfer ratios. Because the two isolators are fabricated in an integrated form their characteristics tend to track well with temperature. As mentioned previously, and not indicated in (2), the desired output offset or bias current is set by a bias potentiometer which adds or subtracts an offset current to the second term of (2) to provide the desired positive output quiescent current.

Fig. 5 shows the near ideal characteristics of the 4-terminal circuit of Fig. 4 over a $1000-\mathrm{V} 150-\mathrm{mA}$ operating region. The first step is the zero-current input step which is set for a quiescent output bias current of about $15 \mathrm{~mA}$. Each incremental input step increases the output current by about $16 \mathrm{~mA}$ giving a current gain of about 80 . These results agree well with the forward current gain of (2) using typical opto-isolator current transfer ratios of about 0.24 . Note the uniformity in the current gain over the 150 $\mathrm{mA}$ range and the sharp current limiting that occurs above $150 \mathrm{~mA}$. The slight upward slope of the characteristic curves represents the output impedance which is mostly due to the gate biasing resistors. The input resistance (terminals 1 and 2) is about $4 \mathrm{k} \Omega$. Thus with an average forward current gain of 80 , the forward transconductance is about $0.02 \mathrm{~S}$.

Fig. 6 shows a complete circuit diagram of the power amplifier. Each polarity output driver is the same as shown in Fig. 4, except an additional $1000 \mathrm{~V}, \mathrm{~N}$-channel MOSFET has been added to provide a $2000-\mathrm{V}$ capability to each polarity driver. The additional MOSFET provides an operating voltage, safety factor of two for each device when operating at maximum peak-to-peak output signal swing. In addition, the total power dissipation for each driver pair is equally divided between two devices. A small trimmer capacitor $C 23$, helps to balance the differential capacitance across the isolator pair so that the highfrequency response for the positive and negative output signal can be better matched. Diodes $C R 3$ and $C R 4$ separate the signal at the output of $U 1$ and steer the respective polarities to each driver. $U 1$ is a high-gain wideband operational-type amplifier that provides the major portion of the open-loop gain for the power amplifier. $U 1$ 's input offset errors are reduced by the loop gain of $U 2$ which in effect servos out any offset errors at the summing junction. In order for this scheme to be effective $U 2$ must be a precision low-offset type of amplifier. A local compensating network $(C 1, R 7)$ around $U 1$ is necessary to shape the gain-bandwidth response to avoid loop oscillation which is inevitable when the control amplifier's gainbandwidth product is dominate in the system. The diodes across the summing junction protect the amplifiers $U 1$ and $U 2$ against high voltages during turn-on and slew-rate limiting.

\section{Performance}

The power amplifier attains its superior output amplitude stability by employing precision, low-temperature coefficient $\left(<1 \mathrm{ppm} /{ }^{\circ} \mathrm{C}\right.$ ) gain determining resistors $R 1$ and $R 2$ in addition to a large open-loop gain. A large overall open-loop gain results from the gain provided by the output driver stage in addition to the high gain of the control amplifier, $U 1$. The closed-loop gain error $\epsilon$, expressed in parts per million ( $\mathrm{ppm}$ ) at frequencies below $1 \mathrm{kHz}$, is shown as

$$
\epsilon=10^{6} /\left(1+\left(A_{0} G_{m} R_{L}\right) /\left(1+A_{c 1}\right)\right)
$$

where $A_{0}$ is the open-loop voltage gain of $U 1, G_{m}$ is the forward transconductance of the output driver, and $A_{c 1}$ is the closed-loop gain $(R 2 / R 1)$. An appreciation for the 


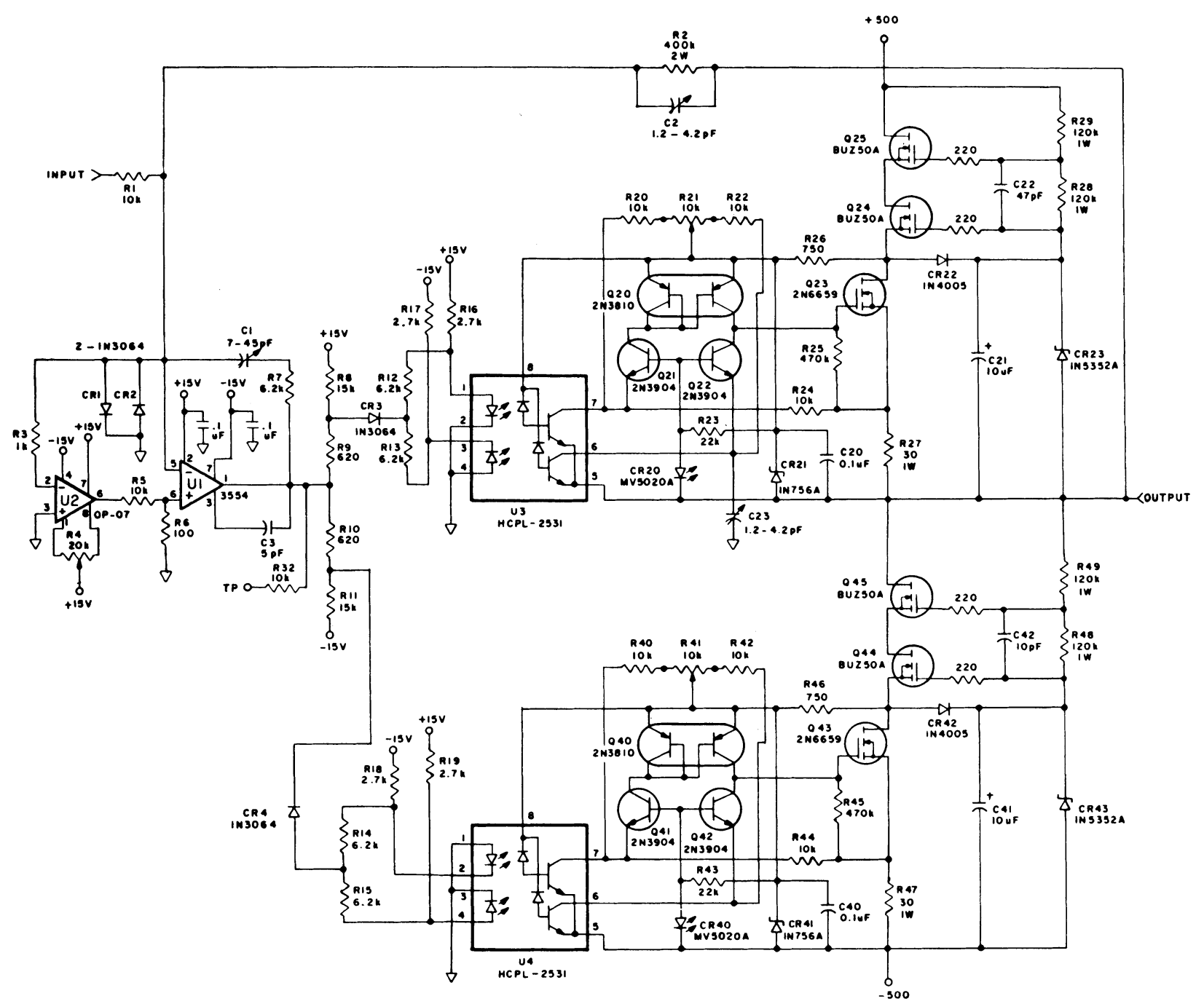

Fig. 6. Complete circuit diagram of the precision power amplifier.

small gain error at a full load current of $100-\mathrm{mA} \mathrm{rms}$ and $120-\mathrm{V}$ rms output (equivalent $R_{L}=1.2 \mathrm{k} \Omega$ ) can be seen by substituting typical values of $10^{5}, 0.02$, and 40 for $A_{0}$, $G_{m}$, and $A_{c 1}$ respectively into (3) which results in an error of $17 \mathrm{ppm}$. This result agrees well with measurements that indicated a change in output amplitude from a "no-load", to a "full-load" condition of about $20 \mathrm{ppm}$ at $60 \mathrm{~Hz}$. Short term (10-min interval) output amplitude stability measurements under "no-load" conditions indicate an instability of $\pm 5 \mathrm{ppm}$. In reality the total load resistance in (3) is the equivalent parallel resistance of the gate biasing resistors and $R 2$ so that the "no-load" resistance for the circuit is about $92 \mathrm{k} \Omega$. At frequencies approximately above $1 \mathrm{kz}$ the loop gain of the system begins to drop off and the gain stability and load regulation performance will drop proportionally. Although extensive stability and load regulation measurements have not been made at higher frequencies to date, a spot measurement at $200-\mathrm{V}$ rms output and $20 \mathrm{kHz}$ indicates only a 0.12 -percent change from no-load to full-load $(2.2 \mathrm{k} \Omega$ or $90-\mathrm{mA} \mathrm{rms})$.

When the power amplifier is used in power calibration applications the stability of the phase shift through the amplifier becomes as important as amplitude stability. The
$150-\mathrm{kHz}$ upper cutoff frequency of the amplifier relative to a $60-\mathrm{Hz}$ operating frequency not only reduces the absolute phase offset but tends to minimize the time-dependent phase instabilities. The short-term phase instability of the amplifier was monitored by using a dual-channel digital generator adjusted to produce a zero power factor $\left(90^{\circ}\right)$ equivalent power source between the power amplifier output and the reference channel. A current-comparator power-bridge was used to indicate phase changes [9]. The results showed a \pm 5 - $\mu$ rad phase instability over a 10 min period.

The maximum output slew rate of the amplifier is about $400 \mathrm{~V} / \mu \mathrm{S}$. Slew-rate limiting in the output stage relates to the ability of the amplifier to charge and discharge output circuit capacitances and limits the full power output frequency response. The total output capacitance $(\simeq 200$ $\mathrm{pF})$ is due to the parallel combination of the MOSFET interelectrode capacitances and the drain-to-heatsink capacitance. Although there is no industry wide value accepted for the distortion level that determines full power response, the amplifier has a near full power output (300V rms@75 mA) response of $50 \mathrm{kHz}$ with total harmonic distortion under 1 percent. 
The average performance characteristics of the power amplifier are summarized:

1) voltage gain: 40 (nom),

2) maximum output swing: $\pm 485 \mathrm{~V},(340-\mathrm{V}$ rms $)$

3) maximum output current: $\pm 145 \mathrm{~mA},(100-\mathrm{mA}$ rms $)$

4) frequency response: dc to $150 \mathrm{kHz}, 3 \mathrm{~dB}(240-\mathrm{V}$ rms 100 -mA output),

5) load regulation: $20 \mathrm{ppm}$ change ( $\mathrm{nl}$ to $\mathrm{fl} @ 120-\mathrm{V}$ rms and $1 \mathrm{kHz}$ ),

6) output noise: $<50-\mathrm{mV}$ rms ( $500 \mathrm{kHz}$ bandwidth),

7) input impedance: $10 \mathrm{k} \Omega$,

8) maximum slew rate: $400 \mathrm{~V} / \mu \mathrm{S}$.

\section{Conclusions}

A precision power amplifier intended primarily for power/energy calibration applications has been described. The design uses high-voltage $N$-channel MOSFET's in the output driver stage together with a unique circuit topology of opto-isolators that provide analog signal coupling and common-mode voltage isolation between the low-level input stage and the high-level output. It was shown that the quiescent operating current of the output driver is essentially independent of the MOSFET parameters, and protected against excessive load currents or short circuits. Although designed for the above specific application, the amplifier's broad range of characteristics should be useful for other applications requiring precision high-voltage power amplification.

\section{ACKNOWLEDGMENT}

The author wishes to thank Robert Palm for his valuable contribution in the fabrication of five complete amplifiers, and Nile Oldham and Keith Lentner for providing extensive performance measurements.

\section{REFERENCES}

[1] N. M. Oldham, O. B. Laug, and B. C. Waltrip, "Digitally-synthesized power calibration source," in IEEE Conf. Precision Electromagnetic Measurements, p. 20, June 1986.

[2] D. F. Stout, M. Kaufman, Handbook of Operational Amplifier Circuit Design. New York: McGraw-Hill, 1976, ch. 23, pp. 7-10.

[3] J. G. Graeme, Application of Operational Amplifiers, Third Generation Techniques. New York: McGraw-Hill, 1973, pp. 40-49.

[4] J. Williams, "High-powered booster circuits enhance op-amp output," EDN, May 27, 1981, pp. 117-124.

[5] - "Boost op-amp output without sacrificing drift and gain specs.," $E D N$, May 29, 1986, vol. 31 , no. 11, pp. 131-144.

[6] - " "Solve oscillation problems when implementing op-amp power booster stages,"' $E D N$, June 12, 1986, vol. 31, no. 12, pp. 203-207.

[7] R. Severns and J. Armijos, Mospower Applications Handbook, Siliconix Inc., 1984, ch. 6, pp. 105-124.

[8] Applications Engineering Staff, Optoelectronics Application Manual. New York: McGraw-Hill, 1977, ch. 3.

[9] N. M. Oldham and O. Petersons, "Calibration of standard wattmeters using a capacitance bridge and digital generator,' IEEE Trans. Instrum. Meas., IM-34, pp. 521-524, Dec. 1985. 